\title{
OBTENÇÃO DE HIDROCARBONETOS SUPERIORES A PARTIR DA CONVERSÃO DO BIOETANOL SOBRE CATALISADORES Ni/ZSM-5
}

\author{
D. LIMA ${ }^{1}$ e O. W. P. LOPEZ ${ }^{1}$ \\ ${ }^{1}$ Universidade Federal do Rio Grande do Sul, Departamento de Engenharia Química \\ E-mail para contato: dirlelima@gmail.com
}

\begin{abstract}
RESUMO - A busca por novas fontes de energia tem levado ao desenvolvimento de pesquisas sobre a produção de hidrocarbonetos a partir do etanol, entre eles os compostos aromáticos. A zeólita ZSM-5 apresenta propriedades adequadas para a obtenção desses compostos e, ainda, propicia que as reações sejam processadas em temperaturas mais amenas. Neste trabalho foram avaliados catalisadores de Ni/ZSM-5 com diferentes teores de níquel, preparados por impregnação com excesso de solvente. Os ensaios foram realizados em um micro-reator tubular de leito fixo. A faixa de temperatura estudada foi de 300-400 ${ }^{\circ} \mathrm{C}$. A análise dos produtos ocorreu por cromatografia gasosa em linha utilizando detectores TCD e FID, e nitrogênio como gás de arraste. Os resultados mostram que todos os catalisadores levaram à conversão completa do etanol, sendo a seletividade para a formação dos hidrocarbonetos superiores influenciada por fatores como: temperatura, teor de Ni impregnado e vazão de etanol na alimentação. Temperaturas no intervalo de $325-350{ }^{\circ} \mathrm{C}$ foram mais favoráveis para a formação desses hidrocarbonetos. Quanto ao teor de $\mathrm{Ni}$ impregnado, obteve-se maior seletividade para os hidrocarbonetos superiores conforme o aumento do teor de Ni no catalisador, sendo mais alta para a amostra com 2,5 \% de Ni. Ainda, foi possível observar que a utilização de $325{ }^{\circ} \mathrm{C}$ como temperatura de reação e baixas vazões de etanol favorece a formação de hidrocarbonetos superiores.
\end{abstract}

\section{INTRODUÇÃO}

O suprimento de energia compõe a base da estruturação e da dinâmica operacional da sociedade humana nos seus mais diversos aspectos. Neste contexto, o petróleo tem tido elevada importância, sendo responsável pelo fornecimento de um terço da energia primária consumida no planeta. Assim, alterações no suprimento ou no uso do petróleo ocasionariam desdobramentos econômicos, políticos e sociais significativos para a maior parte das nações do mundo (SILVA, 2010).

A composição química do petróleo, baseada em hidrocarbonetos de grande heterogeneidade molecular, abre caminhos para usos industriais mais especializados e sofisticados. Por isso, o petróleo é a principal matéria-prima empregada para produzir os derivados utilizados tais como: combustíveis, lubrificantes e produtos petroquímicos (QUELHAS et al., 2011).

Atualmente, devido à diminuição de quantidade e ao aumento do preço desta fonte não renovável de energia, busca-se por fontes renováveis de energia com ênfase no desenvolvimento de processos biotecnológicos que possuam menor impacto ambiental. Nessa busca vêm sendo desenvolvidos processos que permitem a utilização de biomassas para a produção de bioetanol (SILVA, 2010). 
Os hidrocarbonetos aromáticos são obtidos industrialmente através da pirólise da nafta petroquímica ou por reforma catalítica (um dos processos envolvidos no refino de petróleo), assim, a obtenção de hidrocarbonetos (em especial olefinas C3-C4 e aromáticos) através da conversão do bioetanol tem atraído considerável interesse já há algum tempo (GAYUBO et al., $\left.2010^{\mathrm{a}}\right)$.

Trabalhos na literatura mostram que as propriedades da zeólita ZSM-5 são adequadas para a transformação do bioetanol em eteno, C3-C4 olefinas ou em hidrocarbonetos superiores (GAYUBO et al., 2010 ). Experimentos realizados para a reação de conversão de etanol, com diferentes metais impregnados sobre a zeólita ZSM-5, indicam que o rendimento final de produtos BTX é maior que no caso do uso da zeólita pura e, em alguns casos a reação pode ser realizada a temperaturas relativamente mais baixas (MACHADO et al., 2006 e GAYUBO et al., 2010 ).

A acidez e a estrutura porosa das zeólitas desempenham um papel muito importante na desativação dos catalisadores causada pelo coque. Isto não é surpreendente, pois a formação de coque acontece em reações catalisadas por sítios ácidos localizados nos poros. O efeito de desativação das moléculas de coque depende da força dos sítios ácidos, sendo os sítios ácidos mais fortes, aqueles que são mais ativos, os primeiros a se desativarem (GUISNET \& RIBEIRO, 2004). No estudo de Madeira et al. (2010), os resultados mostram que, mesmo que não haja nenhuma desativação observada para a reação de desidratação, existe uma desativação na formação de hidrocarbonetos superiores. É amplamente aceito que a desativação sobre o catalisador ácido sólido começa eliminando os sítios ácidos fortes, de Brönsted, disponíveis. Assim sendo, pode-se supor que a formação de hidrocarbonetos superiores requer sítios ácidos mais fortes do que a reação de desidratação do etanol.

O objetivo desse trabalho é analisar a influência do teor de Ni impregnado na HZSM-5, para a obtenção de hidrocarbonetos superiores, entre eles os aromáticos, através da reação de conversão do bioetanol.

\section{MATERIAIS E MÉTODOS}

\subsection{Catalisadores}

Os catalisadores estudados foram preparados a partir da zeólita ZSM-5 (comercial) na forma ácida e razão $\mathrm{SiO}_{2} / \mathrm{Al}_{2} \mathrm{O}_{3}$ igual a 20, modificada por impregnação com diferentes teores de $\mathrm{Ni}(0,5 ; 1 ; 1,5 ; 2 ; 2,5$ e $3 \%)$.

A impregnação do Ni foi realizada com excesso de solvente, utilizando nitrato de níquel, mantendo a mistura sob agitação constante à temperatura ambiente por um período de 4 horas, com água como solvente. O material foi seco em estufa a $80^{\circ} \mathrm{C}$ e posteriormente calcinado em um reator tubular de quartzo a $600{ }^{\circ} \mathrm{C}$ durante 2 horas com fluxo de ar sintético de $50 \mathrm{ml} / \mathrm{min}$. A zeólita ZSM-5 pura, utilizada em testes para comparação, também foi calcinada utilizando as mesmas condições dos catalisadores impregnados. 


\subsection{Testes de atividade catalítica}

A atividade catalítica das amostras foi avaliada através da reação de conversão do etanol em um micro-reator tubular de vidro, sendo nitrogênio alimentado no mesmo através de controlador de fluxo mássico. Para a alimentação do etanol reagente utilizou-se uma bomba dosadora tipo seringa com controlador de vazão. O catalisador foi disposto em leito fixo, diluído com esferas de vidro de 32 a 42 mesh de diâmetro e sustentado por lã de quartzo. Os produtos obtidos foram analisados através de cromatografia gasosa em linha (Varian 3600cx), empregando-se nitrogênio como gás de arraste e uma coluna empacotada, Porapak Q, cuja temperatura foi mantida em $200{ }^{\circ} \mathrm{C}$ e utilizaram-se detectores de ionização de chama (FID) e de condutividade térmica (TCD). Para o teste catalítico realizado com vazão fixa de $0,4 \mathrm{~mL} / \mathrm{h}$, foram avaliadas as temperaturas na faixa de $300-400{ }^{\circ} \mathrm{C}$ em intervalos de $25^{\circ} \mathrm{C}$. Também, foi realizado um teste com a finalidade de verificar a influência do tempo de residência, variando a vazão do etanol alimentado. Nesse teste as vazões empregadas foram de 0,$2 ; 0,3 ; 0,4 ; 0,8$ e $1,2 \mathrm{~mL} / \mathrm{h}$. Ainda, foi realizado um teste com duração de 10 horas utilizando a vazão de $0,4 \mathrm{~mL} / \mathrm{h}$ e a temperatura de $325^{\circ} \mathrm{C}$ para o catalisador que apresentou melhores resultados na produção de hidrocarbonetos superiores (2,5\% de Ni), a fim de avaliar a estabilidade deste catalisador.

Para analisar os hidrocarbonetos produzidos avaliaram-se os cromatogramas obtidos pelo detector FID e adotou-se uma classificação de hidrocarbonetos em três grupos segundo seu tempo de retenção (Tabela 1).

Tabela 1 - Classificação dos produtos segundo o tempo de retenção na coluna cromatográfica

\begin{tabular}{|c|c|}
\hline Produto & Tempo de Retenção (min) \\
\hline Hidrocarbonetos leves & $\mathbf{t} \leq 1,3($ etanol $)$ \\
\hline Hidrocarbonetos intermediários & $1,3($ etanol $)<t<4$ \\
\hline Hidrocarbonetos pesados & $t \geq 4$ \\
\hline
\end{tabular}

\subsection{Caracterização}

Para verificar modificações na acidez do catalisador foram realizadas análises de dessorção à temperatura programada (TPD) de amônia em um sistema de análise multipropósito SAMP3, fabricado por Termolab Equipamentos Ltda. Realizou-se um pré-tratamento a $100^{\circ} \mathrm{C}$ com passagem de $30 \mathrm{~mL} / \mathrm{min}$ de hélio durante $30 \mathrm{~min}$. A etapa de adsorção de amônia $(30 \mathrm{~mL} / \mathrm{min})$ foi realizada a $100^{\circ} \mathrm{C}$, por um período de $30 \mathrm{~min}$. Antes de se iniciar o aquecimento em si, foi realizada uma purga com passagem de hélio durante $30 \mathrm{~min}$. Então, a temperatura foi elevada até $750{ }^{\circ} \mathrm{C}$ com rampa de $10^{\circ} \mathrm{C} / \mathrm{min}$, sob fluxo de hélio.

Ainda, para estudar o comportamento do catalisador frente à desativação causada pela formação de coque sobre a superfície do mesmo, foram conduzidas análises de oxidação à temperatura programada (TPO) em termobalança (SDT Q600) com aquecimento até $800{ }^{\circ} \mathrm{C}$ e vazão de ar sintético de $100 \mathrm{~mL} / \mathrm{min}$.

\section{RESULTADOS E DISCUSSÃO}

Os resultados dos testes realizados entre 300 e $400{ }^{\circ} \mathrm{C}$ levaram à completa conversão do etanol para todas as amostras de catalisadores. A Figura 1 apresenta a conversão de etanol em 
hidrocarbonetos leves (a), intermediários (b) e pesados (c), conforme estabelecido pela Tabela 1.

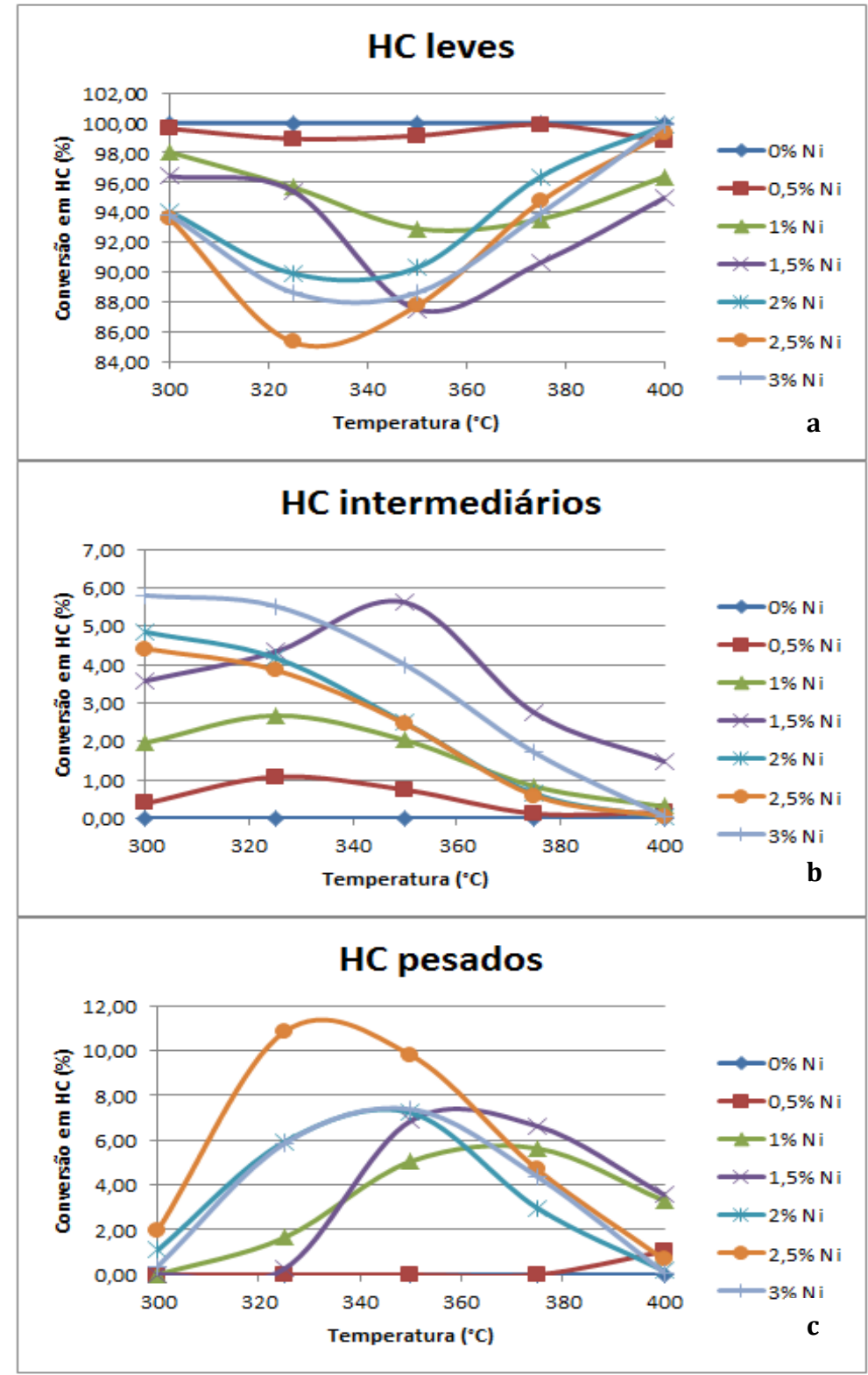

Figura 1 - Produtos da conversão do etanol: (a) HC leves, (b) HC intermediários e (c) HC pesados

A Figura 1a, mostra que quanto menor o teor de Ni impregnado, maior é o percentual de HC leves (principalmente eteno) sendo que menores percentuais desses hidrocarbonetos ocorrem entre $325-350{ }^{\circ} \mathrm{C}$ e para maiores teores de Ni. A Figura 1c, apresenta um comportamento oposto ao da Figura 1a, indicando que o eteno (predominante nos HC leves) é um intermediário para a obtenção dos HC pesados, pois conforme o rendimento desse diminui aumenta o rendimento em HC pesados. Nota-se também, que os catalisadores com menores teores de Ni necessitam de maiores temperaturas para a produção dos HC pesados (produtos de interesse), e que, em temperaturas mais baixas $\left(300^{\circ} \mathrm{C}\right)$ ou muito elevadas $\left(400{ }^{\circ} \mathrm{C}\right)$ a seletividade para esses hidrocarbonetos é baixa, enquanto temperaturas intermediárias (325- 
$350{ }^{\circ} \mathrm{C}$ ) proporcionam maior seletividade. O catalisador que propiciou um maior rendimento nesses hidrocarbonetos foi aquele impregnado com 2,5 \% de níquel, quando a temperatura de reação utilizada foi de $325{ }^{\circ} \mathrm{C}$ (condição que favorece a obtenção dos hidrocarbonetos superiores). Acima de $375{ }^{\circ} \mathrm{C}$ há diminuição, também, dos compostos intermediários e aumento na formação dos compostos leves. A Figura 1b, que trata dos HC intermediários, apresenta um comportamento semelhante ao da Figura 1c, no entanto, nesse caso o catalisador que apresentou um maior rendimento nesses hidrocarbonetos foi aquele impregnado com $1 \%$ de Ni na temperatura de $350{ }^{\circ} \mathrm{C}$.

A Figura 2 apresenta os resultados para o teste realizado com temperatura constante $\left(325^{\circ} \mathrm{C}\right.$ ) e vazão de etanol variável, para o catalisador com $2,5 \%$ de Ni. Esse teste mostrou que a vazão que mais favorece a formação dos hidrocarbonetos superiores é a de $0,4 \mathrm{~mL} / \mathrm{h}$, que já vinha sendo utilizada em testes anteriores, sendo que, no caso de vazões maiores, onde o tempo de contato entre reagente e catalisador é menor, a seletividade para os hidrocarbonetos pesados decaiu, conforme apresenta a Figura 2. Estes resultados mostram a necessidade de um tempo de residência adequado no qual é favorecida a formação de produtos pesados. Tempos de contato curtos favorecem a formação de compostos leves.

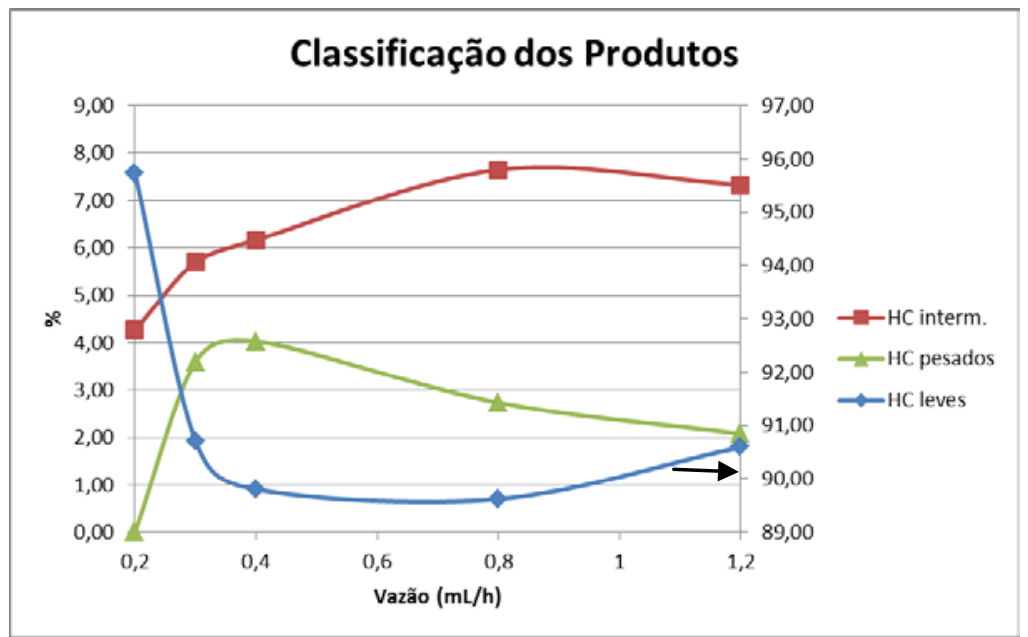

Figura 2 - Influência do tempo de contato entre reagente e catalisador na formação dos produtos

Utilizando as condições que favorecem a obtenção de hidrocarbonetos superiores ( $\mathrm{T}=$ $325{ }^{\circ} \mathrm{C}$ e vazão $=0,4 \mathrm{~mL} / \mathrm{h}$ ), obtidas nos testes realizados anteriormente, foi realizado o teste de desativação para verificar a estabilidade do catalisador impregnado com 2,5 \% de $\mathrm{Ni}$. Os resultados deste teste são apresentados na Figura 3. 


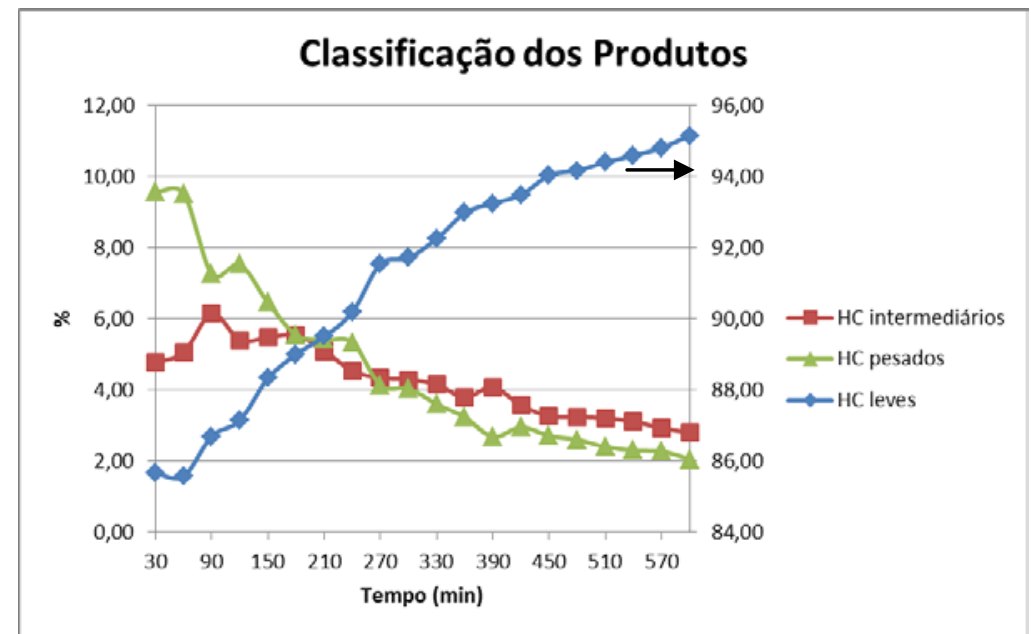

Figura 3 - Influência do tempo de reação na formação dos produtos $\left(\mathrm{T}=325^{\circ} \mathrm{C}\right)$

Observando-se a Figura 3, é possível notar que a seletividade do catalisador para os hidrocarbonetos pesados e intermediários foi decaindo com o aumento do tempo de reação e que ao final de 10 horas predominou a formação de hidrocarbonetos leves. Isto ocorreu devido ao coque formado ao longo da reação que se deposita sobre a superfície do catalisador, bloqueando seus poros dificultando a formação de hidrocarbonetos superiores. Ainda assim, é importante salientar que, mesmo após esse período de 10 horas de reação, houve conversão completa do etanol.

A Figura 4 apresenta um comparativo entre as curvas de TPD-NH3 para a ZSM-5 comercial pura e a amostra impregnada com 2,5\% de Ni.

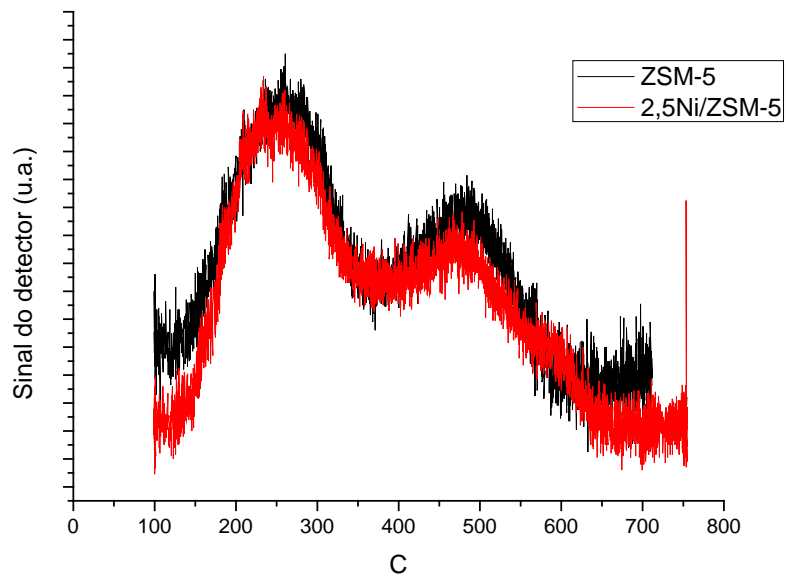

Figura 4 - Influência do Ni na acidez da zeólita ZSM-5 através de TPD-NH 3

Pelas curvas apresentadas na Figura 4, nota-se a presença de um pico em torno de $500{ }^{\circ} \mathrm{C}$ relacionado a acidez forte e um outro pico de maior intensidade em $250{ }^{\circ} \mathrm{C}$ que relaciona-se à quimissorção de $\mathrm{NH}_{3}$ em sítios de acidez moderada. Pode-se observar que o pico relacionado a acidez forte diminui sua área e aparece a uma temperatura mais baixa para a amostra com 2,5\% de Ni, quando comparado à zeólita ZSM-5 pura, indicando uma ligeira diminuição de acidez forte 
do catalisador 2,5 Ni. Este resultado está de acordo com o estudo da natureza dos sítios ácidos de Zhang et al, 2009 que determina que a impregnação da zeólita ZSM-5 com Ni diminui a quantidade de sítios Brönsted (acidez forte).

A Figura 5 apresenta um comparativo entre as curvas de TPO resultantes das análises feitas para cinco dos catalisadores impregnados $(1 ; 1,5 ; 2 ; 2,5$ e 3 \% de Ni), mais a zeólita ZSM-5 pura, que foram utilizados nos testes catalíticos com temperatura na faixa de $300-400{ }^{\circ} \mathrm{C}$ e vazão de $0,4 \mathrm{~mL} / \mathrm{h}$.

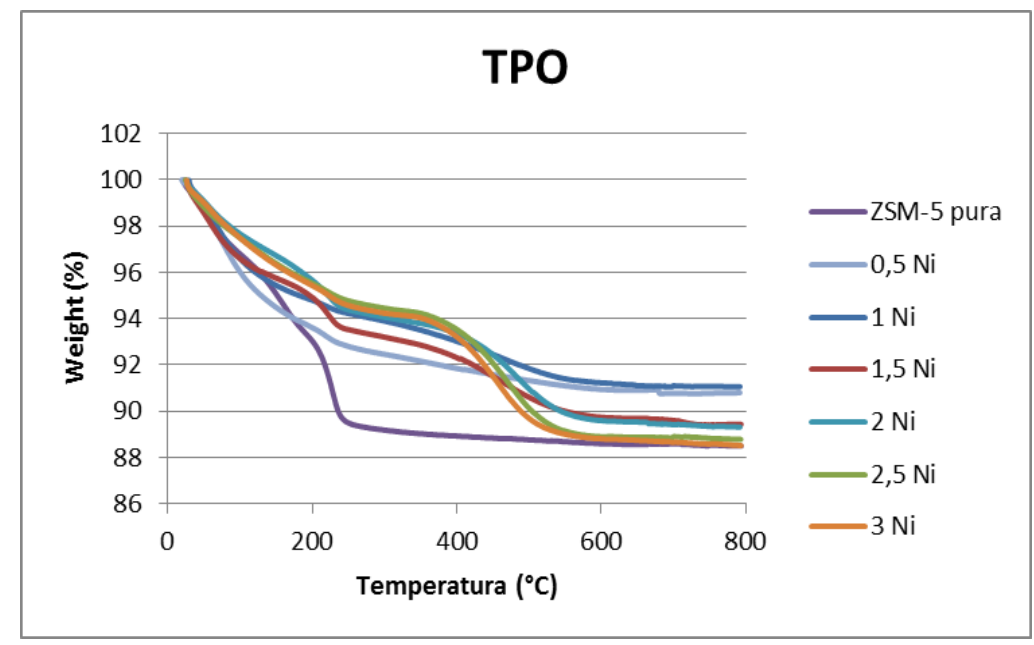

Figura 5 - TPO dos catalisadores após a reação

Nota-se que a maior perda de massa encontrada foi para a ZSM-5 pura, indicando maior formação de coque, o que já era esperado pois a zeólita pura apresenta maior acidez forte que àquelas impregnadas com $\mathrm{Ni}$ e a formação de coque acontece em reações catalisadas por sítios ácidos localizados nos poros. Comparando as amostras impregnadas, nota-se que para maiores teores de $\mathrm{Ni}$, houve maior perda de massa, demonstrando uma maior formação de coque, isso ocorre pois esses catalisadores são aqueles que proporcionam um maior rendimento em HC pesados, sendo que a obtenção desses produtos requer sítios ácidos mais fortes. Também, foi realizada a TPO para a amostra com 2,5 \% de Ni utilizada no teste de estabilidade. Essa análise mostrou que mesmo em um tempo de reação mais longo houve menor formação de coque sobre a superfície do catalisador, quando comparado às TPO's dos catalisadores utilizados nos testes com temperatura variável (300 a $400^{\circ} \mathrm{C}$ ). Isto pode ser explicado pelo fato de o teste de estabilidade ter sido todo realizado na temperatura de $325^{\circ} \mathrm{C}$, evidenciando que em temperaturas mais altas ocorre maior formação de coque e, consequentemente, maior desativação do catalisador.

\section{CONCLUSÃO}

A presença de Ni no catalisador é fundamental para a obtenção de hidrocarbonetos pesados, sendo que a amostra sem Ni (ZSM-5 pura) produziu somente hidrocarbonetos leves, principalmente eteno. Dentre os catalisadores avaliados os melhores resultados foram obtidos para a amostra impregnada com 2,5 \% de níquel. Teores superiores de Ni causam a aglomeração do metal sobre a superfície da zeólita, ao invés de sua dispersão (GAYUBO et al., 2010 ${ }^{\mathrm{b}}$ ), provocando aumento na formação de hidrocarbonetos leves em detrimento dos mais pesados. 
Verificou-se que a temperatura de reação é um parâmetro fundamental que influencia na seletividade do catalisador pois, foi possível notar a existência de um máximo na formação dos hidrocarbonetos superiores para temperaturas intermediárias $\left(325-350{ }^{\circ} \mathrm{C}\right)$.

O teste com diferentes vazões de etanol mostrou que a formação de hidrocarbonetos pesados é favorecida em maiores tempos de residência, isto é, para menores vazões de etanol (entre 0,3 e $0,4 \mathrm{~mL} / \mathrm{h}$ ).

O teste do tempo de campanha mostrou que a seletividade para hidrocarbonetos pesados diminui ao longo da reação em razão da formação de coque sobre à superfície do catalisador.

\section{REFERÊNCIAS}

GAYUBO, A.G.; ALONSO, A.; VALLE, B.; AGUAYO, A.T.; BILBAO, J. Selective production of olefins from bioethanol on HZSM-5 zeolite catalysts treated with $\mathrm{NaOH}$. Appl. Catal., B, v.97, p.299-306, 2010 .

GAYUBO, A.G.; ALONSO, A.; VALlE, B.; AGUAYO, A.T.; OLAZAR, M.; BILBAO, J. Hydrothermal stability of HZSM-5 catalysts modified with $\mathrm{Ni}$ for the transformation of bioethanol into hydrocarbons. Fuel, v.89, p.3365-3372, 2010

GUINSNET, M.; RIBEIRO, F. R. Zeólitos: um nanomundo ao serviço da catálise. Edição da Fundação Calouste Gulbenkian. Lisboa. 2004.

MACHADO, N.R.; CALSAVARA, V.; ASTRATH, N.G.; NETO, A.M.; BAESSO, M.L. Hydrocarbons from ethanol using [Fe,Al]ZSM-5 zeolites obtained by direct synthesis. Appl. Catal., A, v.311 p.193-198, 2006.

MADEIRA, F.F.; GNEP, N.S.; MAGNOUX, P.; VEZIN, H.; MAURY, S.; CADRAN N. Mechanistic insights on the ethanol transformation into hydrocarbons over HZSM-5 zeolite. Chem. Eng. J., v.161 p.403-408, 2010.

QUELHAS, A.D.; PASSOS, C.N.; LAGE, D.F.S.; ABADIE, E.; SOUSA, E.C.M.; CORDEIRO, F.G.; KRAHL, I.M.; FARAH, M.A.; ARAÚJO, M.A.S.; BRASIL, N.I.; CAMARGO, P.R.C.; PINTO, R.R.C. Processamento de Petróleo e Gás: Petróleo e seus derivados, processamento primário, processos de refino, petroquímica, meio ambiente. Rio de Janeiro: LTC, 2011.

SILVA, N.L.C. Produção de bioetanol de segunda geração a partir de biomassa residual da indústria de celulose. 2010. 123 f. Dissertação (Mestrado em Tecnologia de Processos Químicos e Bioquímicos) - Universidade Federal do Rio de Janeiro, Escola de Química, Rio de Janeiro, 2010.

ZHANG, X.; ZHONG, J.; WANG, J.; ZHANG, L.; GAO, J.; LIU, A. Catalytic performance and characterization of Ni-doped HZSM-5 catalysts for selective trimerization of n-butene. Fuel Process. Technol., v.90 p.863-870, 2009. 